\title{
Eficacia de las etiquetas de advertencia en las cajetillas de cigarrillo
}

\author{
Carlos Gantiva, ${ }^{1}$ Stefanny Palacio, ${ }^{1}$ Alejandra Ortega, ${ }^{1}$ Karen Castillo ${ }^{1}$ \\ y Katherine Ortiz ${ }^{1}$
}

Forma de citar

Gantiva C, Palacio S, Ortega A, Castillo K, Ortiz K. Eficacia de las etiquetas de advertencia en las cajetillas de cigarrillos. Rev Panam Salud Publica. 2018;42:e101. https://doi.org/10.26633/RPSP.2018.101

RESUMEN Objetivo. Establecer la capacidad que tienen las etiquetas de advertencia con el tamaño mínimo exigido por la Organización Mundial de la Salud para capturar la atención en fumadores y no fumadores.

Métodos. En el estudio se incluyeron 30 fumadores y 30 no fumadores, quienes realizaron una prueba de detección del punto (dot-probe) en la cual observaron, de manera simultánea, imágenes de cajetillas de cigarrillo divididas en dos: la parte superior contenía la marca de los cigarrillos y la parte inferior contenía la etiqueta de advertencia. Durante la tarea, se registró la actividad cerebral a través de los potenciales relacionados a eventos del tipo potencial negativo que ocurre aproximadamente a los $200 \mathrm{~ms}$ en la zona posterior contralateral al estímulo (N2pc) y negatividad contralateral posterior sostenida (SPCN, por sus siglas en inglés), los cuales son indicadores de atención temprana y sostenida.

Resultados. En ambos grupos se encontró mayor amplitud de los potenciales N2pc y SPCN hacia la porción de la cajetilla que contiene la marca de cigarrillos. Sin embargo, el tiempo de reacción fue menor ante la etiqueta de advertencia en la tarea dot-probe.

Conclusiones. Los resultados sugieren que, al inicio, la atención se dirige hacia la marca de los cigarrillos y luego hacia la etiqueta de advertencia. La incapacidad de las etiquetas de advertencia para capturar la atención en las primeras fases disminuye su eficacia, sobre todo en fumadores. Se sugiere aumentar el tamaño de las etiquetas de advertencia para favorecer un incremento en la respuesta de atención.

Palabras clave Tabaco; advertencia; política de salud; etiquetado de productos; atención.

A nivel mundial, el consumo de tabaco es responsable de más de siete millones de muertes al año (seis millones o más son consumidores directos y alrededor de 890000 son no fumadores

\footnotetext{
Laboratorio de Neurociencias y Neuropsicología, Departamento de Psicología, Universidad de San Buenaventura, Bogotá, Colombia. Enviar la correspondencia a Carlos Gantiva, cgantiva@gmail.com
}

expuestos al humo ajeno). También tiene una relación directa con la aparición de 29 enfermedades, es la principal causa de cáncer de pulmón y es responsable de más de $50 \%$ de las enfermedades cardiovasculares (1).

Para disminuir el consumo de tabaco y desmotivar su inicio, en el año 2005 la Organización Mundial de la Salud
(OMS) desarrolló el Convenio Marco para el Control del Tabaco (CMCT). Desde entonces, ha sido suscrito por 168 países que representan $86,5 \%$ de los países en el mundo $(2,3)$. Uno de los mecanismos más utilizados en el CMCT para advertir los peligros del consumo de tabaco es el uso de etiquetas de advertencia en los empaques de cigarrillo. En la

Este es un artículo de acceso abierto distribuido bajo los términos de la licencia Creative Commons Attribution-NonCommercial-NoDerivs 3.0 IGO, que permite su uso, distribución y reproducción en cualquier medio, siempre que el trabajo original se cite de la manera adecuada. No se permiten modificaciones a los artículos ni su uso comercial. Al reproducir un artículo no debe haber ningún indicio de que la OPS o el artículo avalan a una organización o un producto específico. El uso del logo de la OPS no está permitido. Esta leyenda debe conservarse, junto con la URL original del artículo. 
actualidad, 135 países utilizan etiquetas de advertencia conformadas por imágenes y textos (1).

Las investigaciones relacionadas con las etiquetas de advertencia muestran que son más efectivas en personas que no han iniciado el consumo, en quienes lo iniciaron recientemente y en los fumadores que están motivados para abandonarlo (4-7). Se ha identificado que las etiquetas que usan imágenes son más efectivas que aquellas que solo usan texto (8), y que las imágenes de advertencia generan un impacto emocional aversivo con un nivel medio de intensidad $(9,10)$. Hoy en día, 57 países utilizan etiquetas de advertencia con el tamaño mínimo exigido por la OMS (1) (30\% del empaque), aunque la evidencia muestra que el impacto emocional disminuye de manera significativa con este tamaño (11).

Debido a que la atención es necesaria para el procesamiento cognitivo y emocional del mensaje (12), uno de los principales requisitos para asegurar la eficacia de las etiquetas de advertencia es que estas sean capaces de capturar la atención (13). Esto se debe a que la etiqueta de advertencia debe competir con el porcentaje de la cajetilla que ocupan la marca, el logotipo y los colores de la compañía de tabaco. Debido a la proximidad en tiempo y espacio con el comportamiento de consumo, estos elementos se convierten en estímulos relevantes desde el punto de vista motivacional por su asociación con el cigarrillo, lo cual genera un sesgo atencional ante dichos estímulos (14) y una respuesta de deseo de consumo (craving) y de búsqueda del consumo de tabaco (15).

Las investigaciones que han estudiado la respuesta atencional ante las etiquetas de advertencia lo han hecho a través de la tarea dot-probe (16). Estas investigaciones muestran que, en fumadores ligeros, los empaques con etiquetas compuestas por imágenes que ocupan más del 30\% del empaque generan mayor sesgo atencional (17) y que las etiquetas de advertencia compuestas por imagen y texto generan mayor sesgo atencional en fumadores en comparación con los no fumadores (18).

Sin embargo, el uso de la tarea dot-probe como único indicador de sesgo atencional ha sido controversial, debido a que solo mide un sesgo atencional tardío y a la poca confiabilidad interna que ha demostrado tener (19-21). Por lo anterior, el uso de técnicas electrofisiológicas como la electroencefalografía (EEG) y los potenciales relacionados a eventos (PRE), se sugieren como indicadores más confiables, que complementan la tarea del dot-probe, debido a su óptima resolución temporal.

Los PRE N2pc y SPCN han demostrado medir de manera confiable el sesgo atencional temprano y la atención sostenida, respectivamente. El N2pc es un potencial negativo que ocurre aproximadamente a los $200 \mathrm{~ms}$ en la zona posterior contralateral al estímulo (21). La negatividad contralateral posterior sostenida $(\mathrm{SPCN})$ es también un potencial negativo que emerge aproximadamente a los 400 ms después del inicio del estímulo y es máximo en la región occipito-parietal del hemisferio contralateral al estímulo atendido (22). La integración de la tarea dot-probe con el $\mathrm{N} 2$ pc y la SPCN permite el análisis del curso temporal de la respuesta de atención $(20,23)$ desde una primera fase de atención focalizada, a través del potencial N2pc, una segunda fase de atención sostenida a través del potencial SPCN y una tercera fase de respuesta atencional tardía a través de la prueba dot-probe.

A través de esta metodología, el objetivo del presente estudio fue evaluar la capacidad que tienen las etiquetas de advertencia que ocupan el 30\% del empaque de cigarrillos para capturar la atención focalizada, sostenida y tardía en fumadores y en no fumadores.

\section{MATERIALES Y MÉTODOS}

\section{Diseño del estudio}

Se llevó a cabo un estudio cuasiexperimental con un diseño factorial mixto $2 \times 2$. Como variable independiente intersujeto se utilizó el grupo (no fumador y fumador) y, como variable independiente intrasujeto, la imagen (marca de los cigarrillos y etiqueta de advertencia). Las variables dependientes fueron la amplitud del N2pc y del SPCN y el tiempo de reacción en la tarea dot-probe.

\section{Participantes}

A través de un muestreo por conveniencia, en el estudio participaron 60 estudiantes de una universidad ubicada en Bogotá, Colombia, con un rango de edad entre 18 y 35 años, distribuidos en dos grupos: fumadores y no fumadores. La muestra se reclutó a través de información escrita y verbal entregada en la biblioteca y los salones. Para ambos grupos, los participantes debían ser mayores de edad (> 18 años). El grupo de fumadores ( $n=30,15$ hombres y 15 mujeres, media $=22,9$ años $\pm 3,8$ ) debía haber fumado más de 100 cigarrillos en su vida (24), fumar al menos cinco cigarrillos diarios y tener una historia de consumo de al menos un año. En promedio, los participantes de este grupo fumaban 6,21 $\pm 1,69$ cigarrillos por día. Ninguno de los participantes del grupo de fumadores estaba interesado en abandonar el consumo de cigarrillo.

El grupo de no fumadores $(n=30$, 16 hombres y 14 mujeres, media $=23,2$ años $\pm 4,4$ ) nunca fumaron ni probaron el cigarrillo. No hubo diferencias significativas en la edad ni en la distribución de los sexos entre ambos grupos $(P>0,75$ en ambos casos). Todos los participantes recibieron información sobre las consecuencias del consumo de cigarrillo y estrategias para prevenirlo o abandonarlo después de la sesión experimental.

\section{Estímulos y tarea experimental}

Se utilizaron 12 imágenes de cajetillas de tres de las marcas de cigarrillos más consumidas en Colombia. Cada cajetilla tenía una etiqueta de advertencia que cubría el 30\% del empaque, según lo estipulado por la ley colombiana (Resolución 3961 de 2009) y el mínimo exigido por la OMS (2). Para evitar el efecto de habituación, se utilizaron etiquetas de los años 2009, 2011, 2012 y 2013, tres de cada año. Cada una de las imágenes se editaron en el programa Photoshop (Adobe Photoshop $^{\circledR}$ ) y se dividieron exactamente por la mitad: el 50\% superior que contiene la marca de los cigarrillos y el 50\% inferior en donde está la etiqueta de advertencia (figura 1). Todas las imágenes se presentaron a color sobre un fondo negro, con un tamaño de $200 \times 160$ píxeles en una pantalla plana de 19 pulgadas localizada a $60 \mathrm{~cm}$ del participante.

La tarea de dot-probe se programó en el software E-Prime 2.0 $0^{\circledR}$ (Psychology Software Tools, PA, Estados Unidos de América). Cada ensayo inició con una cruz de fijación (500 ms), luego cada par de imágenes (el 50\% superior y el 50\% inferior de la cajetilla) aparecían en cada extremo horizontal de la pantalla durante 700 ms. Por último, en uno de los lugares en donde estaba una de las imágenes aparecía un punto. El participante debía oprimir una tecla según el lugar donde 
FIGURA 1. Ejemplos de los estímulos y de la tarea dot-probe utilizada en el experimento

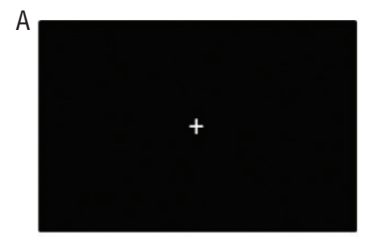

$500 \mathrm{~ms}$

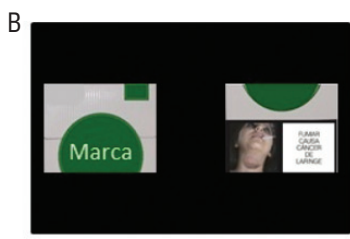

$700 \mathrm{~ms}$

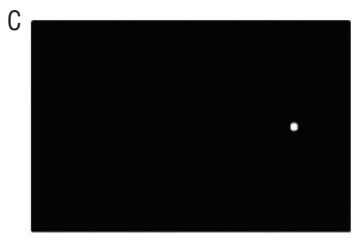

$2000 \mathrm{~ms}$

ms, milisegundos.

A, primera pantalla: cruz de fijación durante $500 \mathrm{~ms}$; B, segunda pantalla: marca de cigarrillos y etiqueta de advertencia durante $700 \mathrm{~ms}$; C, tercera pantalla: punto durante $2000 \mathrm{~ms}$ o hasta que el participante responda. Fuente: Elaboración propia.

estaba ubicado el punto. El límite de tiempo para emitir la respuesta era 2000 $\mathrm{ms}$. El intervalo entre ensayos variaba de forma aleatoria entre 5000 a $6000 \mathrm{~ms}$. Un menor tiempo de reacción indica mayor sesgo atencional.

Cada par de imágenes se presentó cuatro veces: la etiqueta de advertencia a la derecha y cajetilla a la izquierda o viceversa, y para cada una de estas dos alternativas el punto puede aparecer en uno de los dos lados de la pantalla. Estas cuatro posibles combinaciones se presentaron de forma aleatoria. Se realizaron 15 ensayos de prueba con imágenes neutrales del Sistema Internacional de Imágenes Afectivas (IAPS por sus siglas en inglés) $(25,26)$. En total, la tarea tuvo 63 ensayos (15 de prueba y 48 de evaluación).

\section{Registro y procesamiento del electroencefalograma}

Se registró la actividad electroencefalográfica (EEG) de forma continua a través de un equipo BioSemi ActiveTwo de 32 electrodos $^{\circledR}$ (BioSemi, Ámsterdam, Países Bajos) ubicados según el sistema 10/20 con una tasa de muestreo de 1024 Hz. Cada canal se referenció al promedio de la actividad de los 32 canales y se mantuvo la impedancia por debajo de $20 \mathrm{k} \Omega$. Para identificar los parpadeos y movimientos oculares, se registró el electrooculograma (EOG) a través de un electrodo ubicado a un centímetro debajo del ojo derecho y otro ubicado a un centímetro del canto del ojo izquierdo.

La señal del EEG se procesó en Matlab a través de la aplicación EEGLab ${ }^{\circledR}$ (27) y de scripts diseñados específicamente para el estudio. Al comienzo, se disminuyó la tasa de muestreo a $256 \mathrm{~Hz}$ y se aplicó un filtro de banda entre 0,1 y $40 \mathrm{~Hz}$. Se segmentó el registro en períodos de 800 ms
(100 ms antes del estímulo y 700 ms después del estímulo) y se corrigieron los artefactos producto de los movimientos oculares a través del algoritmo de Gratton, Coles y Donchin (28). Se identificaron los valores extremos $(+/-70 \mu \mathrm{V})$ en cada canal y se rechazaron los ensayos que tuvieran cuatro o más canales con estos valores: en total, se eliminó el 5,1\% de los ensayos.

El registro de los potenciales evocados N2pc y SPCN se hizo en la zona occipitoparietal y contralateral a la ubicación del estímulo que captura la atención (22): por lo tanto, se registraron ambos potenciales en los electrodos PO3 y PO4. Para el N2pc, la ventana de tiempo fue entre $125 \mathrm{~ms}$ y $250 \mathrm{~ms}$ posestímulo y, para el SPCN, entre 400 ms y 700 ms posestímulo. Las amplitudes de los potenciales N2pc y SPCN se calcularon, al inicio, a partir de la actividad del hemisferio contralateral e ipsilateral al estímulo que se deseaba analizar. Por ejemplo, cuando la etiqueta de advertencia aparecía en el extremo derecho de la pantalla, se calculó la actividad en el hemisferio izquierdo (contralateral) y luego la actividad en el hemisferio derecho (ipsilateral). Por último, los potenciales evocados N2pc y SPCN se obtienen de restar la actividad ipsilateral de la contralateral (19-22).

\section{Análisis estadístico}

Los datos de la tarea dot-probe y de los potenciales evocados N2pc y SPCN se analizaron a través de un análisis de varianza (ANOVA por sus siglas en inglés) mixta de medidas repetidas $2 \times 2$, con el grupo (fumador y no fumador) como factor intersujeto y la imagen (marca de cigarrillos y etiqueta de advertencia) como factor intrasujeto. Se aplicó la corrección de Greenhouse-Geisser en todos los casos para corregir cualquier violación de la esfericidad en los datos. La comparación de pares post hoc se hizo con la corrección de Bonferroni para disminuir la probabilidad de error tipo 1 . El nivel de significancia para todos los análisis fue de $P<0,05$ y se reporta el tamaño del efecto $\left(\eta_{\mathrm{p}}{ }^{2}\right.$; pequeño $\geq 0,01$ medio $\geq 0,06$ grande $\geq 0,14)$ (29). Todos los análisis se realizaron en el programa estadístico SPSS $20.0^{\circledR}$.

La investigación fue aprobada por el Comité de Ética de la Universidad de San Buenaventura (Bogotá, Colombia). El consentimiento informado de los participantes se obtuvo por escrito luego de entregar información detallada (escrita y verbal) del procedimiento y objetivo de la investigación. Toda la información fue codificada para garantizar el anonimato de los participantes y se almacenó en computadores con acceso seguro del Laboratorio de Neurociencias y Neuropsicología de la Universidad de San Buenaventura, Bogotá, Colombia.

\section{RESULTADOS}

\section{N2pc}

En la figura 2 se muestra el PRE N2pc en fumadores y no fumadores ante la marca de cigarrillos y la etiqueta de advertencia. Solo la marca de cigarrillos generó una actividad negativa alrededor de los $200 \mathrm{~ms}$, lo que indica un sesgo atencional temprano hacia la marca. Este resultado se corrobora por medio de análisis estadísticos (cuadro 1). El ANOVA reveló un efecto principal significativo para la imagen, la marca de cigarrillos generó una mayor amplitud (mayor negatividad) del potencial N2pc. Se encontró un efecto principal significativo para el grupo, los no fumadores tuvieron mayor amplitud del potencial N2pc en comparación con los fumadores. El efecto de interacción no fue significativo $(P=0,12)$.

\section{SPCN}

La figura 2 también muestra el PRE SPCN en fumadores y no fumadores ante la marca de cigarrillos y la etiqueta de advertencia. Una vez más, la marca de cigarrillos fue la única que generó una actividad negativa entre los 400 y $700 \mathrm{~ms}$, lo que indica el sostenimiento de la atención hacia la marca. Este resultado se corrobora con el análisis estadístico (cuadro 1). El ANOVA identificó un efecto 
principal significativo para la imagen, la marca generó una mayor amplitud del potencial SPCN en comparación con la etiqueta de advertencia. Se encontró un efecto principal significativo para el grupo, los no fumadores tuvieron mayor amplitud del potencial SPCN en comparación con los fumadores. El efecto de interacción no fue significativo $(P=0,50)$.

\section{Tarea de dot-probe}

En el se muestran los tiempos de reacción en la tarea dot-probe en fumadores y no fumadores ante la marca de cigarrillos y la etiqueta de advertencia. El ANOVA reveló un efecto principal significativo para la imagen; el tiempo de reacción ante la etiqueta de advertencia fue significativamente menor, lo que sugiere una respuesta de atención tardía hacia la etiqueta. No se encontró ningún otro efecto principal ni de interacción significativos (ambas $P>0,71$ ).

\section{DISCUSIÓN}

El presente estudio investigó los sesgos atencionales ante las etiquetas de advertencia en los empaques de cigarrillo en fumadores y no fumadores, a través de una metodología que integra medidas

FIGURA 2. Gran promedio de los potenciales relacionados a eventos N2pc y SPCN ante la etiqueta de advertencia y la marca de cigarrillos en no fumadores y fumadores
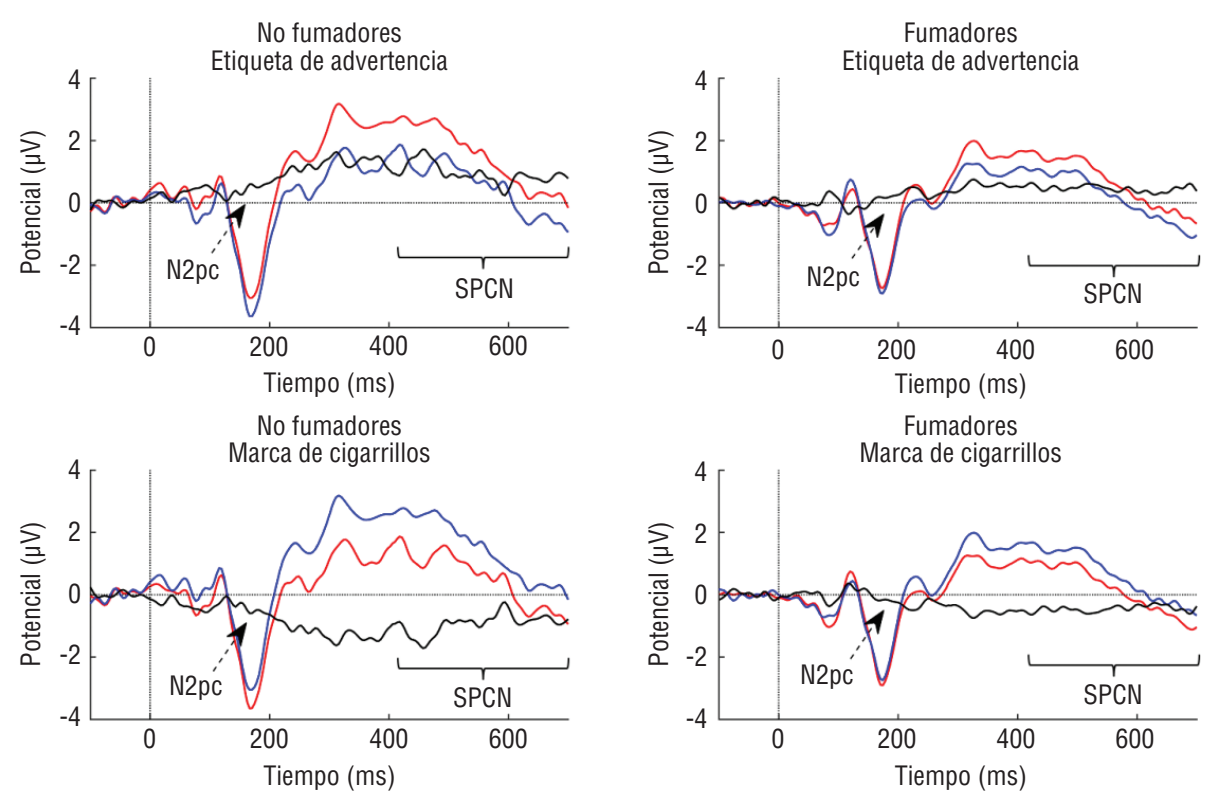

- Contralateral —- Ipsilateral - N2pc/SPCN

SPCN, negatividad contralateral posterior sostenida (por sus siglas en inglés); ms, milisegundos; $\mu \mathrm{V}$, microvoltios. Fuente: Elaboración propia a partir de los resultados presentados.

conductuales (es decir, los tiempos de reacción) y fisiológicas (es decir, los PRE) de la atención. Los resultados indican que la respuesta de atención cambia en el curso del tiempo, al principio se dirige y mantiene hacia la parte de la cajetilla que contiene la marca de cigarrillos y, por último, se redirecciona hacia la etiqueta de advertencia. Se encontró también mayor amplitud de los PRE en no fumadores en comparación con los fumadores, lo que indica, en general, mayor respuesta en las primeras etapas de la atención en los no fumadores.

Las investigaciones previas sobre la respuesta de atención hacia las etiquetas de advertencia han mostrado que, en fumadores, las etiquetas compuestas por imágenes y texto generan mayor atención que aquellas que solo tienen imágenes (18) y el efecto es mayor en fumadores ligeros (17). Sin embargo, estos estudios tienen dos limitaciones, la primera es que solo utilizaron la tarea dot-probe como medida de atención, la segunda, es que en ambas investigaciones no se comparó el sesgo atencional ante los componentes de la cajetilla de cigarrillos (es decir, la parte que posee la marca versus la parte que posee la etiqueta de advertencia). Por lo tanto, no pudieron determinar cuál parte de la cajetilla es la que genera una mayor respuesta atencional.

Los resultados de la presente investigación sugieren que, en un primer momento, la atención es capturada por la parte de la cajetilla que posee el nombre, colores y logotipo que identifican a la marca de cigarrillos. Esta primera respuesta atencional ocurre en forma temprana, aproximadamente a los 150-250 ms, y corresponde con la mayor amplitud del PRE N2pc, el cual ha demostrado ser un indicador confiable del primer sesgo atencional ante estímulos relevantes

CUADRO 1. Amplitud de los potenciales relacionados a eventos y de los tiempos de reacción en la prueba dot-probe ante la marca de cigarrillos y la etiqueta de advertencia en fumadores y no fumadores

\begin{tabular}{|c|c|c|c|c|c|c|}
\hline & Marca de cigarrillos & Etiqueta de advertencia & $F$ & gl & Valor de $P$ & $\eta_{p}{ }^{2}$ \\
\hline $\mathrm{N} 2 \mathrm{pc}(\mu \mathrm{V})$ & $-1,59 \pm 1,40$ & $-0,65 \pm 1,24$ & 7,31 & 1,47 & 0,01 & 0,13 \\
\hline $\operatorname{SPCN}(\mu \mathrm{V})$ & $-2,45 \pm 2,42$ & $-1,04 \pm 1,76$ & 6,77 & 1,47 & 0,01 & 0,12 \\
\hline Tiempo de reacción en la tarea dot-probe (ms) & $537,6 \pm 107,09$ & $523,8 \pm 90,29$ & 7,49 & 1,46 & 0,009 & 0,14 \\
\hline $\mathrm{N} 2 \mathrm{pc}(\mu \mathrm{V})$ & $-1,27 \pm 0,50$ & $-0,97 \pm 0,40$ & 5,53 & 1,47 & 0,02 & 0,10 \\
\hline $\operatorname{SPCN}(\mu \mathrm{V})$ & $-2,19 \pm 1,19$ & $-1,31 \pm 0,43$ & 12,35 & 1,47 & 0,001 & 0,20 \\
\hline
\end{tabular}

$\mathrm{DE}$, desviación estándar; $\mu \mathrm{V}$, microvoltios; ms; milisegundos; NS, no significativo; $\mathrm{F}$ test (análisis de varianza); gl, grados de libertad; $\eta_{p}^{2}$, eta parcial al cuadrado (tamaño del efecto). Datos presentados como promedio $\pm \mathrm{DE}$.

Fuente: Elaboración propia a partir de los resultados presentados. 
desde el punto de vista motivacional $(19,20)$. Esta primera respuesta atencional es sostenida hasta después de los $400 \mathrm{~ms}$ y hasta los $700 \mathrm{~ms}$ aproximadamente, tiempo en el cual se observa mayor amplitud del potencial SPCN, el cual es un indicador confiable de atención sostenida y permite el mantenimiento de la información visual en la memoria en el corto plazo (22-30). En conjunto, estos resultados indican que, en un primer momento, la etiqueta de advertencia con un tamaño del 30\% del empaque de cigarrillos no logra capturar los recursos atencionales.

La incapacidad de las etiquetas de advertencia de generar una respuesta atencional en una fase inicial, puede generar en los fumadores una respuesta de craving y de búsqueda del consumo de tabaco. Esto se debe a que el porcentaje de la cajetilla que posee la marca y los logotipos ha adquirido en los fumadores saliencia incentiva por la asociación que ha tenido con el cigarrillo $(14,15)$. Lo anterior sugiere que el uso de etiquetas de advertencia en los empaques de tabaco es una política de salud pública que tiene menor impacto en los fumadores.

Luego de la fase inicial de la respuesta atencional, los resultados indican que después de un segundo la atención se dirige hacia la etiqueta de advertencia. De esta forma, se observa un cambio en el foco atencional, del porcentaje de la cajetilla con la marca y logotipo de los cigarrillos hacia la etiqueta de advertencia. Este resultado puede llegar a modular la respuesta de craving en los fumadores (14). Sin embargo, los más beneficiados serán los no fumadores ya que ellos, al no experimentar ningún tipo de respuesta de ansia por el consumo en la fase inicial (15) y, luego, dirigir su atención hacia la etiqueta de advertencia, podrán procesar mejor su mensaje tanto desde el punto de vista emocional como cognitivo.

En conjunto, los resultados del presente estudio sugieren que las etiquetas de advertencia que tienen el tamaño mínimo exigido por la OMS (2) no son capaces de generar un sesgo atencional temprano en fumadores y no fumadores, sino que solo logran captar la atención después de haber transcurrido un segundo, lo que disminuye su eficacia como estrategia de control del consumo de tabaco.

Con respecto a las limitaciones del estudio, en primer lugar, este se desarrolló solo con participantes fumadores y con no fumadores. No se tienen datos sobre población exfumadora, lo que permitiría establecer la eficacia de las etiquetas de advertencia como estrategia de salud pública para la prevención de recaídas. En segundo lugar, el grupo de fumadores estuvo conformado por fumadores diarios, deberán desarrollarse futuras investigaciones en fumadores ocasionales. En tercer lugar, de los criterios de los Centros para el Control y la Prevención de Enfermedades (CDC por sus siglas en inglés) (31), el grupo de fumadores puede ser considerado como ligeros (entre 5 y 14 cigarrillos al día). Por lo tanto, se aconseja realizar investigaciones con fumadores medios (15 a 24 cigarrillos diarios) y pesados (más de 25 cigarrillos al día). Sin embargo, el grupo de fumadores tiene una historia de consumo de $15,96 \pm 2,66$ meses en promedio, lo que indica el desarrollo del hábito tabáquico y establece una clara diferencia con el grupo de no fumadores, que reportaron nunca haber consumido cigarrillo. Por otra parte, no se midió el nivel de dependencia a la nicotina, lo que puede influir en la respuesta atencional hacia los estímulos asociados al cigarrillo. No obstante, el consumo diario de cigarrillo y la historia de consumo han mostrado ser factores determinantes en las respuestas condicionadas a los estímulos asociados al tabaco (32). Por último, el estudio se desarrolló con etiquetas de advertencia que ocupaban el 30\% de la cajetilla, futuros estudios deberán determinar el tamaño ideal para que las etiquetas generen un sesgo atencional temprano.

\section{CONCLUSIÓN}

Al integrar los datos de los PRE y de la tarea dot-probe, los resultados indican que las etiquetas de advertencia solo logran capturar la atención de fumadores y no fumadores después de un segundo, aproximadamente, lo que disminuye su eficacia, sobre todo en los fumadores. Los resultados también sugieren que el tamaño mínimo exigido por la OMS para las etiquetas de advertencia no es suficiente para generar un sesgo atencional inicial, lo que dificulta su posterior procesamiento emocional y cognitivo. A partir de lo anterior, el aumento en el tamaño de la etiqueta de advertencia podría ser una estrategia eficaz para el control del consumo de tabaco.

Financiamiento. Esta investigación se realizó con recursos de la Universidad de San Buenaventura, sede Bogotá (Ref. CHS 012-010).

Conflicto de intereses. Ninguno declarado por los autores.

Declaración. Las opiniones expresadas en este manuscrito son responsabilidad del autor y no reflejan necesariamente los criterios ni la política de la RPSP/ PAJPH y/o de la OPS.

\section{REFERENCIAS}

1. Organización Mundial de la Salud (OMS). WHO report on the global tobacco epidemic, 2015. Raising taxes on tobacco. Ginebra: OMS; 2015. Disponible en: http:/ / www.who.int/tobacco/global_ report/2015/report/en/

2. Organización Mundial de la Salud (OMS). El Convenio Marco de la OMS para el Control del Tabaco. Ginebra: OMS; 2005. Disponible en: http:/ / www.who.int/fctc/ text_download/es/

3. Organización Mundial de la Salud (OMS). MPOWER un plan de medidas para hacer retroceder la epidemia de tabaquismo. Ginebra: OMS; 2008. Disponible en: http://www.who.int/tobacco/mpower/package/es/

4. Hammond D, Fong G, McNeill A, Borland R, Cummings K. Effectiveness of cigarette warning labels in informing smokers about the risks of smoking: findings from the International Tobacco Control (ITC) Four Country Survey. Tob Control. 2006;15(S3):iii19-iii25. doi:10.1136/tc.2005. 012294

5. Hammond D, Reid JL, Driezen P, Boudreau C. Pictorial health warnings on cigarette packs in the United States: an experimental evaluation of the proposed FDA warnings. Nicotine Tob Res. 2013;15(1):93-102. doi $10.1093 /$ ntr/nts094

6. Hammond D, Thrasher J, Reid JL, Driezen P, Boudreau C, Santillán EA. Perceived effectiveness of pictorial health warnings among Mexican youth and adults: a population-level intervention with potential to reduce tobacco-related inequities. Cancer Causes Control. 2012;23(1):57-67. doi: 10.1007/s10552-012-9902-4

7. Thrasher JF, Arillo-Santillán E, Villalobos V, Pérez-Hernández R, Hammond D, Carter J, et al. Can pictorial warning labels 
on cigarette packages address smoking-related health disparities? Field experiments in Mexico to assess pictorial warning label content. Cancer Causes Control. 2012;23(1): 69-80. doi: $10.1007 /$ s10552-012-9899-8

8. Noar SM, Hall MG, Francis DB, Ribisl KM, Pepper JK, Brewer NT. Pictorial cigarette pack warnings: a meta-analysis of experimental studies. Tob Control. 2015;25:341-54. doi:10.1136/tobaccocontrol-2014-051978

9. Muñoz MA, Viedma-del-Jesús MI, Rosselló F, Sanchez-Nacher N, Montoya P, Vila J. The emotional impact of European tobaccowarning images. Tob Control. 2011;22(2): 123-29. doi:10.1136/tobaccocontrol-2011050070

10. Nascimento BE, Oliveira L, Vieira AS, Joffily M, Gleiser S, Pereira MG, et al. Avoidance of smoking: the impact of warning labels in Brazil. Tob Control. 2008;17(6):405-09. doi:10.1136/tc.2008.025643

11. Gantiva C, Guerrero L, Rico A, Ramírez N, Díaz M, González M, Romo-González T. Influence of cigarette package brand on the emotional impact of tobacco-warning images that cover $30 \%$ of cigarette packs in smokers and nonsmokers. Nicotine Tob Res. 2016;18(5):1324-30. doi:10.1093/ntr/ ntv257

12. Wogalter MS, Vigilante WJ. Attention switch and maintenance. En: Wogalter MS editor. Handbook of Warnings. Mahwah, NJ/ London: Lawrence Erlbaum Associates; 2006.

13. Argo JJ, Main KJ. Meta-analyses of the effectiveness of warning labels. J Public Policy Mark. 2004;23(2):193-208. doi: 10.1509/jppm.23.2.193.51400

14. Ehrman RN, Robbins SJ, Bromwell MA, Lankford ME, Monterosso JR, O'Brien CP. Comparing attentional bias to smoking cues in current smokers, former smokers, and non-smokers using a dot-probe task. Drug Alcohol Depend. 2002;67(2):185-91. doi: 10.1016/S0376-8716(02)00065-0

15. Berridge KC. Wanting and liking: Observations from the neuroscience and psychology laboratory. Inquiry. 2009;52(4): 378-98. doi:10.1080/00201740903087359

16. MacLeod C, Mathews A, Tata P. Attentional bias in emotional disorders. J Abnorm Psychol. 1986;95(1):15-20.

17. Loeber S, Vollstädt-Klein S, Wilden S, Schneider S, Rockenbach C, Dinter C, et al. The effect of pictorial warnings on cigarette packages on attentional bias of smokers. Pharmacol Biochem Behav. 2011;98(2): 292-98. doi: 10.1016/j.pbb.2011.01.010

18. Brown KG, Reidy JG, Weighall AR, Arden MA. Graphic imagery is not sufficient for increased attention to cigarette warnings: the role of text captions. Addiction. 2013; 108(4):820-25. doi:10.1111/add.12008

19. Kappenman ES, Farrens JL, Luck SJ, Proudfit GH. Behavioral and ERP measures of attentional bias to threat in the dot-probe task: poor reliability and lack of correlation with anxiety. Front Psychol. 2014;5. doi: 10.3389/fpsyg.2014.01368

20. Kappenman ES, MacNamara A, Proudfit $\mathrm{GH}$. Electrocortical evidence for rapid allocation of attention to threat in the dot-probe task. Soc Cogn Affect Neurosci. 2015; 10(4):577-83. doi: $10.1093 /$ scan/nsu098

21. Xu M, Li Z, Diao L, Fan L, Yang D. Contextual valence and sociality jointly influence the early and later stages of neutral face processing. Front Psychol. 2016;7:1258. doi: 10.3389/fpsyg.2016.01258

22. Gibb BE, Pollak SD, Hajcak G, Owens M. Attentional biases in children of depressed mothers: An event-related potential (ERP) study. J Abnorm Psychol. 2016;125(8): 1166-78. doi: 10.1037 /abn0000216

23. Usher M, McClelland JL. The time course of perceptual choice: the leaky, competing accumulator model. Psychol Rev. 2001; 108(3):550-92. doi: 10.1037/0033-295X.108. 3.550

24. Centers for Disease Control and Prevention. Vital signs: current cigarette smoking among adults aged $\geq 18$ years, United States, 2005-2010. MMWR. 2011;60(35): 1207.
25. Lang P, Bradley MM, Cuthbert BN. International affective picture system (IAPS): affective ratings of pictures and instruction manual (Technical Report A-8). Gainesville, FL: University of Florida; 2008.

26. Gantiva C, Guerra P, Vila J. Validación colombiana del sistema internacional de imágenes afectivas: evidencias del origen transcultural de la emoción. Acta Colomb Psicol. 2011;14(2):103-11.

27. Delorme A, Makeig S. EEGLAB: an open source toolbox for analysis of single-trial EEG dynamics including independent component analysis. J Neurosci Methods. 2004;134(1):9-21. doi: 10.1016/j.jneumeth. 2003.10.009

28. Gratton G, Coles MG. Donchin E. A new method for offline removal of ocular artifact. Electroencephalogr Clin Neurophysiol. 1983;55:468-84.

29. Cohen J. Statistical power analysis for the behavioral sciences (2nd ed.). Hillsdale, NJ: Erlbaum; 1988.

30. Jolicoeur P, Brisson B, Robitaille N. Dissociation of the N2pc and sustained posterior contralateral negativity in a choice response task. Brain Res. 2008;1215: 160-72. doi: 10.1016/j.brainres.2008.03.059

31. Maurice E, Trosclair A, Merritt R, Caraballo R, Malarcher A, Husten C, et al. Cigarette smoking among adults, United States, 2004. JAMA. 2006;295(7):749-51. doi:10.1001/jama.295.7.749

32. Cui Y, Robinson JD, Versace F, Lam CY, Minnix JA, Karam-Hage $M$, et al. Differential cigarette-related startle cue reactivity among light, moderate, and heavy smokers. Addict Behav. 2012;37(8): 885-9. doi:10.1016/j.addbeh.2012.02.003

Manuscrito recibido el 12 de diciembre de 2017. Aceptado para su publicación, tras revisión, el 29 de marzo de 2018. 
ABSTRACT Objective. To determine the ability of cigarette-pack warning labels, of the minimum size required by the World Health Organization, to capture the attention of smokers and nonsmokers.

Effectiveness of cigarettepack warning labels in capturing the attention of smokers and nonsmokers

Methods. In this study, 30 smokers and 30 nonsmokers completed a dot-probe task in which they simultaneously observed images of cigarette packs split in two: the top contained the cigarette brand and the bottom contained the warning label. During the task, brain activity was recorded through two event-related potentials of the negative-polarity type--the potential that occurs in the posterior-contralateral zone approximately $200 \mathrm{~ms}$ after a stimulus (N2pc) and the sustained posterior contralateral negativity $(\mathrm{SPCN})$ response-which are indicators of early and sustained attention.

Results. In both groups, a greater amplitude of N2pc and SPCN potentials was found for the part of the pack containing the cigarette brand. However, during the dot-probe task, reaction times were shorter for the warning label.

Conclusions. These results suggest that, initially, attention is focused on the cigarette brand, and only then on the warning label. The inability of warning labels to capture early-phase attention decreases their effectiveness, especially in smokers. We suggest that warning labels be enlarged to facilitate increased attention response.

Keywords Tobacco; warning; health policy; product labeling; attention.

RESUMO

Eficácia das tarjas de advertência em maços de cigarro para atrair a atenção de fumantes e não fumantes

Objetivo. Determinar a capacidade das tarjas de advertência com o tamanho mínimo exigido pela Organização Mundial da Saúde (OMS) de atrair a atenção de fumantes e não fumantes.

Métodos. A amostra do estudo consistiu de 30 fumantes e 30 não fumantes que foram testados com uma tarefa de sondagem (dot-probe task) em que tiveram de observar simultaneamente imagens de um maço de cigarro dividido ao meio: a parte superior continha a marca do cigarro e a parte inferior continha a tarja de advertência. Durante o teste, foi registrada a atividade cerebral com a análise de potenciais evocados relacionados a eventos do tipo potencial negativo que ocorre em cerca de $200 \mathrm{~ms}$ na área posterior contralateral ao estímulo (N2pc) e da ocorrência de negatividade contralateral posterior sustentada (SPCN, em inglês), que são indicadores de atenção mais rápida e sustentada.

Resultados. Em ambos os grupos de participantes foi observada maior amplitude dos potenciais N2pc e SPCN na parte do maço que continha a marca. Porém, o tempo de reação na tarefa de sondagem foi menor diante da tarja de advertência.

Conclusões. Os resultados deste estudo sugerem que, ao início, a atenção é dirigida à marca do cigarro e depois à tarja de advertência. A incapacidade da tarja de advertência de atrair a atenção nas primeiras fases diminui sua eficácia, sobretudo entre os fumantes. Recomenda-se aumentar o tamanho das tarjas de advertência para estimular um incremento na resposta de atenção.

Palavras-chave

Tabaco; aviso; política de saúde; rotulagem de productos; atenção. 